\title{
Quantitative Orientation-Independent Differential Interference Contrast (DIC) Microscopy Coupled with Orientation-Independent Polarization Microscopy
}

\author{
Michael Shribak ${ }^{*}$, James LaFountain ${ }^{* *}$, David Biggs ${ }^{* * *}$, and Shinya Inoué \\ *Marine Biological Laboratory, Woods Hole, MA, USA 02543 \\ ** Dept. Biological Sciences, University at Buffalo, Buffalo, NY, USA 14260 \\ **** AutoQuant Imaging, Inc., 105 Jordan Road, Troy, NY, USA 12180
}

Differential interference contrast (DIC) microscopy is widely used to observe structure and motion in unstained, transparent living cells and isolated organelles, producing a monochromatic shadowcast image of optical phase gradient. Polarized light microscopy (Pol) reveals structural anisotropy due to form birefringence, intrinsic birefringence, stress birefringence, etc. DIC and Pol complement each other as, for example, in a live dividing cell, the DIC image will clearly show the chromosomes while the Pol image will depict the distribution of the birefringent microtubules in the spindle. Both methods, however, have the same shortcomings: they require the proper orientation of a specimen in relation to the optical system in order to achieve best results.

We describe here orientation-independent differential interference contrast (OI-DIC) microscopy, which records phase gradients within microscopic specimens independently of their orientation [1]. OI-DIC generates images representing the distribution of optical phase in the specimen, i.e., high fidelity interference microscope images. Unlike in other forms of interference microscopes, OI-DIC does not require a narrow illuminating cone. This new system is probably one of the few, if not the only, microscope system, which allows the generation of interference microscope images at highest NAs.

The feasibility of OI-DIC was demonstrated with various specimens such as glass rods embedded in Permount, Siemens star nano-fabricated in $90-\mathrm{nm}$ thick silicon oxide layer, Bovine pulmonary artery endothelial cell, human cheek squamous epithelial cell, Sciara coprophila salivary gland polytene chromosomes, Vorticella convallaria, crane-fly (Nephrotoma suturalis) spermatocytes, etc, using regular DIC optics on a microscope equipped with a precision rotating stage. Several conventional DIC images were recorded with the specimen oriented in different directions, but with the prism bias unchanged, followed by digital alignment and processing of the images. Then the obtained images are used for computation of the phase gradient magnitude and azimuth distribution, and, further, the phase image. OI-DIC data can also be used to generate enhanced, regular DIC images with any desired shear direction. The results demonstrate that OI-DIC, which does not require re-orientation of the specimen, can successfully image and measure phase gradients and phase of transparent specimens, independent of the direction of the gradients.

The OI-DIC system can also be combined with an orientation-independent polarization (OI-Pol) system $[2,3]$. The combined system will yield two complementary phase images of thin optical sections of the specimen: distribution of refractive index gradient and distribution of birefringence due to structural or internal anisotropy of the cell structure. For instance, in a live dividing cell, the OI-DIC image will clearly show detailed shape of the chromosomes while the polarization image will quantitatively depict the distribution of the birefringent microtubules in the spindle. 
Figure 1 is an example of color combination of OI-DIC and OI-Pol images of a crane-fly spermatocyte at metaphase of meiosis I, containing a group of 4 conventional DIC images (left top) and a group of 4 conventional polarization images (left bottom). These raw images were employed for computing phase (dry mass) and retardance images (middle top and bottom accordingly). On the far right is a color combination of the phase (dry mass) and retardance modes, in which red and green colors correspond to dry mass distribution and retardance, respectively. Morphological structures, such as chromosomes, are especially prominent in the phase mode image. The birefringent spindle fibers (actually bundles of microtubules) exhibit much better contrast in the retardance mode. The phase image acquired in this fashion yields the true distribution of optical path and optical anisotropy using 1.4 NA optics.

\section{References}

[1] M. Shribak, and S. Inoué, "Orientation-independent differential interference contrast microscopy", Applied Optics, 45: 460-469 (2006).

[2] R. Oldenbourg, and G. Mei, "New polarized light microscope with precision universal compensator." Journal of Microscopy, 180, 140-147 (1995).

[3] M. Shribak, and R. Oldenbourg, "Technique for fast and sensitive measurements of twodimensional birefringence distribution", Applied Optics, 42: 3009-3017 (2003).

[4] The encouragement and support of Dr. Rudolf Oldenbourg of the Marine Biological Laboratory are gratefully acknowledged.

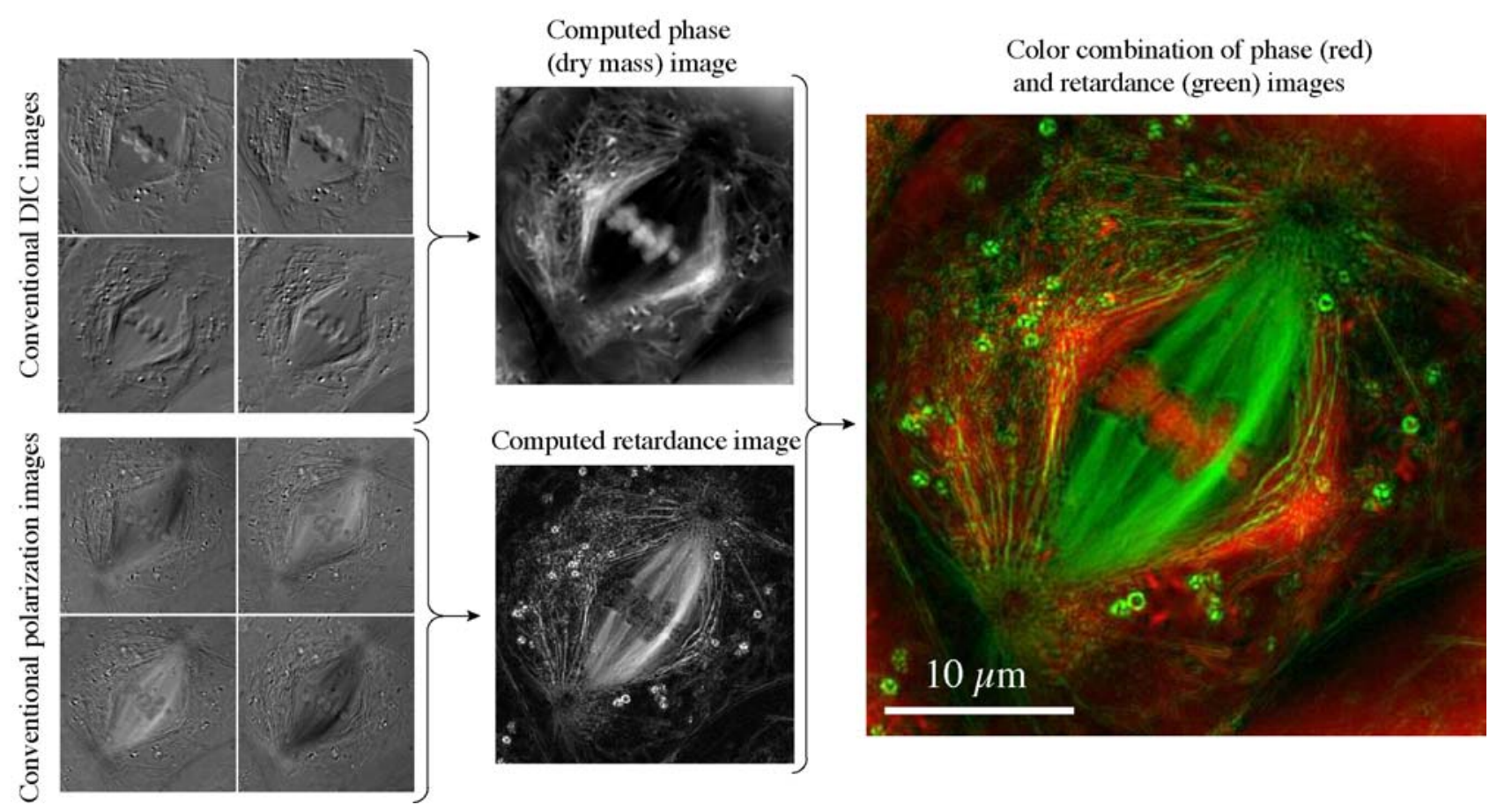

Figure 1. Metaphase of meiosis I in a crane-fly spermatocyte. 